\title{
1 ZIELSETZUNG, THEMATISCHER HINTERGRUND UND BERICHTSAUFBAU
}

\section{$1.1 \quad$ Projektziele}

Übergeordnetes Projektziel: Das übergeordnete Ziel des Projekts war die partizipative Entwicklung von Konzepten und Instrumenten für eine Integration von Wildtieren, Wildtierlebensräumen und der Möglichkeit einer nachhaltigen Jagdausübung in ein sektorübergreifend abgestimmtes nachhaltiges Wildtiermanagement und in eine gesamt nachhaltige regionale Landnutzung im Biosphärenpark Wienerwald. Dafür sollten Prinzipien, Kriterien und Indikatoren für integratives nachhaltiges Wildtiermanagement im Biosphärenpark Wienerwald entwickelt werden, die auf nachhaltigkeitsrelevante Schnittstellen zwischen Wildtieren, deren Lebensräumen, deren Bejagung und den Einflüssen unterschiedlicher regionaler Landnutzergruppen fokussieren. Die Bewertungssets sollen der Selbstüberprüfung jener Landnutzer dienen, die maßgebliche Einflüsse auf die Erhaltung von heimischen Wildtierarten und deren Lebensräumen ausüben. Dabei waren Konflikt- und Synergiepotenziale zwischen einer nachhaltigen Jagdausübung und anderen regionalen Landnutzungen zu berücksichtigen.

Abgeleitete Teilziele: Zur Erreichung des übergeordneten Projektzieles sollte die Erfüllung folgender Teilziele beitragen:

- Entwicklung regionsbezogener Prinzipien, Kriterien und Indikatoren für eine nachhaltige Jagd bzw. für jagdliche Aktivitäten im Biosphärenpark Wienerwald unter Einbeziehung von Nachhaltigkeitsanforderungen an andere Landnutzungssektoren (Vermeidung von Wildschäden in der Land- und Forstwirtschaft, Erhaltung der Artenvielfalt, etc.).

- Identifizierung und Analyse zentraler Schnittstellen zwischen Wildtieren und deren Lebensräumen, einer nachhaltigen Jagdausübung und den Aktivitäten weiterer Landnutzergruppen im Wildtierlebensraum.

- Entwicklung von Prinzipien, Kriterien und Indikatoren für wesentliche nichtjagdliche Landnutzergruppen im Hinblick auf ein integratives (sektorübergreifendes) nachhaltiges Wildtiermanagement im Biosphärenpark Wienerwald,

- zur Nachhaltigkeitsbewertung der Aktivitäten von Landnutzergruppen im Hinblick auf die nachhaltige Erhaltung heimischer Wildarten und ihrer Lebensräume sowie auf eine nachhaltige Jagdausübung,

- zur Selbstüberprüfung durch regionale Landnutzergruppen mit Einfluss auf Wildtiere, Lebensräume und die Nachhaltigkeit der Jagd.

- Ableitung von Empfehlungen für ein integratives Wildtier- und Habitatmanagement sowie für ein diesbezügliches Nachhaltigkeitsmonitoring im Biosphärenpark Wienerwald.

- Bereitstellung von Projektergebnissen, die zur Erfüllung der Erhaltungs- und Entwicklungsziele des Biosphärenparks Wienerwald sowie zu dessen Management beitragen, und die auch in anderen Biosphärenparks angewendet werden können.

Ausgehend von bestehenden allgemeinen, d. h. österreichweit anwendbaren Bewertungskriterien für nachhaltige Jagd, die ausschließlich eine Bewertung von jagdlichen Aktivitäten ermöglichen (Forstner et al., 2001; 2003; 2006), sollte im vorliegenden Projekt der weiterführende Entwicklungsschritt zur integralen Bewertungsmöglichkeit der Aktivitäten unterschiedlicher Landnutzer im Hinblick auf die Auswirkungen dieser Aktivitäten auf die Nachhaltigkeit der Jagd und des Wildtiermanagements erfolgen; es sollte eine "GesamtNachhaltigkeitsbewertung“ zum Thema „Wildtiere, Wildtierlebensräume und Jagd“ entwickelt werden. Bestehende Nachhaltigkeitskriterien für den Landnutzungssektor „Jagd“ sollten da- 
bei an den Biosphärenpark Wienerwald angepasst und durch vergleichbare Nachhaltigkeitskriterien für die Nutzungssektoren Landwirtschaft, Forstwirtschaft sowie Freizeit- und Erholungsnutzung erweitert werden. Dadurch sollte erstmals eine sektorübergreifend abgestimmte Nachhaltigkeitsbeurteilung geschaffen werden. Der Biosphärenpark Wienerwald diente dabei als Modellregion.

Wesentliche Schnittstellen - im Sinne von nachhaltigkeitsrelevanten Interaktionsfeldern, Antagonismen und Synergismen - zwischen Wildtieren, Wildtierlebensräumen und wildtierrelevanten Landnutzungssektoren sollten identifiziert und daraufhin analysiert werden, inwieweit diese Sektoren zur Sicherung einer nachhaltigen Jagd und zur Erhaltung von Wildtierpopulationen und -lebensräumen eingebunden werden müssen.

Ausgehend vom übergeordneten Ziel der Einbindung von Wildtieren und der Jagd in eine gesamthaft nachhaltige Landnutzung, die auch den Anforderungen des Naturschutzes entspricht, sollten die Begriffe "Integratives nachhaltiges Wildtiermanagement" und "Nachhaltige Jagd" (einschließlich der Erhaltung von Wildtierpopulationen und deren Lebensräumen) mit einem Bewertungsset von Prinzipien, Kriterien und Indikatoren inhaltlich konkretisiert und dadurch mit Leben erfüllt werden, wobei ökologische, ökonomische und soziokulturelle Aspekte zu berücksichtigen waren. Aus diesen Ergebnissen waren Empfehlungen für ein entsprechend integrales und nachhaltiges Management sowie für ein Nachhaltigkeitsmonitoring abzuleiten.

Im Projekt sollte ein transdisziplinärer Arbeitsansatz angewandt werden, in dem regionale Stakeholder und Landnutzer eng in das Projekt eingebunden werden, und in dem ein breites Spektrum von partizipativen Forschungsmethoden eingesetzt wird.

\subsection{Thematischer Hintergrund}

\section{Problemlage: Wildtiere als komplexes „Querschnittsthema“}

Einerseits leistet nachhaltige Jagd für die Gesellschaft wertvolle Beiträge, indem sie z. B. qualitativ hochwertiges Wildbret als Nahrungsmittel auf den Markt bringt, Problemwildarten (Wildschäden, Krankheitsübertragung etc.) reguliert und Biotopverbesserungen durchführt. Nachhaltige Jagd kann zur Vermeidung land- und forstwirtschaftlicher Schäden und zur Erhaltung gefährdeter Tier- und Pflanzenarten sowie traditioneller Kulturlandschaften und Bräuche maßgeblich beitragen.

Andererseits scheint die Jagd den Interessen nichtjagdlicher Gesellschaftsgruppen immer häufiger entgegen zu stehen, und es ergeben sich zum Teil sehr gegensätzlich ausgerichtete Interessen und Bedürfnisse. Zu Konflikten kommt es insbesondere dann, wenn durch Wildtiere privates oder öffentliches Gut beschädigt wird oder aber jagdfremde Personen infolge der Jagdausübung in der Ausübung ihrer Tätigkeiten beeinträchtigt werden, z. B. ihren Drang nach „Freiheit“ und „Wildnis“ nicht ausleben können. Zu starken Konflikten kommt es außerdem, wenn der Jäger durch ungünstige Umstände (die durch andere Interessensgruppen mitverschuldet sein können) die Schalenwilddichte nicht den lokalen Gegebenheiten anpassen (regulieren) kann. Waldbesitzer fürchten in diesem Fall um ihre Bäume und um die Verjüngung der Wälder; Landwirte laufen Gefahr, einen Teil ihrer Ernte einzubüßen; und Natur- und Artenschützer sehen nicht ein, weshalb z. B. durch überhöhte Wildschweinbestände der Naturwert seltener Orchideenwiesen aufs Spiel gesetzt wird. Tierfreunde und Tierschützer wiederum zeigen oft mangelndes Verständnis, warum Wildtierbestände in der Kulturlandschaft reguliert und fallweise stark reduziert werden müssen.

Neben forst- und landwirtschaftlicher Nutzung hat der Biosphärenpark Wienerwald - gerade durch seine Lage zur Millionenstadt Wien - große Bedeutung für die Freizeit- und Erholungsnutzung. Die Bevölkerung sieht in der „Grünen Lunge Wiens“ (seit der legendären 
Rettung des Wienerwaldes durch Schöffel Ende des 19. Jahrhunderts) hohes Erholungsund gesundheitliches Regenerationspotenzial. Die Jagd und der allgemeine (bewusste und unbewusste) Umgang mit Wildtieren (Schutz, Beobachtung, Habitatveränderungen, Stressfaktoren, etc.) ist - neben der Nutzung von Luft - praktisch die einzige flächendeckende Form von Ressourcennutzung. Wildtiere tangieren zahlreiche Nutzergruppen und sind deshalb ein "Querschnittsthema“, das leicht zur Polarisierung bestehender Interessen führen kann. Besonders leicht erfolgt dies im Hinblick auf die Freizeit- und Erholungsnutzung, die dem flächendeckenden Charakter von Jagd und Wildtiermanagement zumindest im großstadtnahen Biosphärenpark Wienerwald wohl am nächsten kommt. Als Folge dieser unterschiedlichen Nutzungsansprüche auf gleicher Fläche und oft auch zur gleichen Zeit resultieren Flächennutzungskonflikte, die oft zu Ungunsten von Wildtieren und deren Lebensräumen sowie der Möglichkeit einer nachhaltigen Jagdausübung ausgehen, gleichzeitig aber auch die Nutzungsqualität anderer beteiligter Landnutzungsansprüche beeinträchtigen können. Wildlebensräume werden meist stetig und oft lange Zeit unbemerkt kleiner und zunehmend fragmentierter, weshalb sich die Problemlage dann rasch zuspitzen kann. [Mit der Fragmentierung und notwendigen Vernetzungskonzepten im Biosphärenpark Wienerwald befasst sich das Projektteam im Rahmen eines neuen MAB-Projekts ab Herbst 2008].

Am Beispiel des ökologisch, ökonomisch und sozio-kulturell komplexen und sensiblen Themas „Wildtiere, Wildtierlebensräume und jagdliche Nutzung" lässt sich die sektorübergreifende, d. h. landnutzergruppenübergreifende Abhängigkeits- und Ursachenverflechtung gut erkennen. Es zeigt sich hier deutlich, dass für einen bestimmten Nutzungssektor (in diesem Fall die Jagd) eine Nutzung überhaupt nur dann nachhaltig möglich sein kann (auch wenn der Nutzungssektor selbst die Nachhaltigkeitsanforderungen erfüllt), wenn eine ausreichende Abstimmung der Nachhaltigkeitskriterien mit anderen Nutzungssektoren erfolgt. Ein isoliertes, sektorales Nachhaltigkeitskonzept, das lediglich auf das Handlungsfeld der Jagd und auf jagdliche Einflüsse des Wildtiermanagements ausgerichtet ist, kann weder die Nutzungsform "Jagd“ bzw. eine Möglichkeit zur Jagdausübung noch die nachhaltige Erhaltung von Wildtierarten, Wildpopulationen und Wildlebensräumen nachhaltig sichern.

Das vielseitig vernetzte Querschnittsthema „Wildtier“ birgt aber nicht nur ein erhebliches Konfliktpotenzial in sich, sondern auch das Potenzial eines verbindenden Elements, weil viele Menschen sich davon berührt fühlen. Deshalb wurde dieses Themenfeld im vorliegenden Projekt als Modellbeispiel für die Entwicklung einer themenbezogenen, sektorübergreifenden Gesamtnachhaltigkeit aufgegriffen. Gerade die gesellschaftliche Abstimmung zwischen den verschiedenen Funktionen von Biosphärenparks (siehe Kapitel 2.4) erfordert ein hohes Maß intersektoraler Kooperation. Generelles Anwendungsziel soll die Minimierung von Reibungsverlusten und die langfristige, d. h. nachhaltige Etablierung von Problemlösungskonzepten (präventiv und kurativ) sein. Natürliche Ressourcen sind ein kostbares Gut geworden, und zu ihrer Bewahrung bedarf es gemeinsamer Anstrengungen. In vielen Fällen decken sich „grüne“ Interessen von Forstleuten, Landwirten, Naturschützern und Jägern ohnedies, sodass Synergieeffekte erzielbar sind (sofern die Zusammenhänge und Wechselwirkungen bekannt sind). Für die möglichst konfliktfreie Abstimmung der Wienerwald(be)nutzung (im konkreten Fall in Bezug auf das Wildtiermanagement) soll das intersektorale Zusammenspiel lokaler Interessengruppen über ein GesamtNachhaltigkeitskonzept optimiert werden, um dadurch zu einer insgesamt nachhaltigen Regionalentwicklung sowie zur Vermeidung von Konflikten und wechselseitigen Beeinträchtigungen der verschiedenen Landnutzer beizutragen.

\section{Kontext mit Nachhaltigkeitskonzept}

Durch die Hege und Entnahme von frei lebenden Wildtieren beeinflusst die Jagd einen Teil der natürlichen Ressourcen. Dadurch werden unmittelbar die genetische Vielfalt einzelner 
Wildarten, die Wildartenzusammensetzung und Struktur von Wildtierpopulationen beeinflusst, auf mittelbarem Wege aber auch nicht jagdbare Tierarten sowie Pflanzenarten und Boden. Dieser Einfluss kann Auswirkungen auf Ökosysteme haben und birgt Konfliktpotenzial mit anderen Nutzern natürlicher Ressourcen (z. B. der Forstwirtschaft, der Landwirtschaft, Freizeit- und Erholungsnutzungen). Wildtiere, deren Vorkommen und Verhalten sowie deren Bejagbarkeit können auch von Änderungen der Landnutzung, von Infrastruktureinrichtungen (z. B. Straßen, Bahn- od. Leitungstrassen) und anderen anthropogenen Faktoren stark beeinflusst werden. Die vielfältigen „außerjagdlichen“ Einflussfaktoren auf Wildtiere, deren Lebensräume und Bejagbarkeit, welche die Möglichkeiten einer nachhaltigen Jagd oft stark einschränken, werden im Rahmen des vorliegenden Projektberichts weitest möglich analysiert und bewertet. Der Projektbericht befasst sich somit, wie oben erwähnt, nicht nur mit den Auswirkungen jagdlicher Aktivitäten, sondern auch mit den Auswirkungen von Landund Forstwirtschaft sowie Freizeit- und Erholungsmanagement auf eine nachhaltige Entwicklung von Wildtieren, Wildtierlebensräumen und Jagd. Der primäre Fokus liegt auf denjenigen wild lebenden Tierarten, die aufgrund der jeweiligen Jagdgesetze in den Zuständigkeitsbereich der Jagd fallen, einschließlich der ganzjährig geschonten Wildarten, sowie auf seltenen bzw. gefährdeten Arten.

„Nachhaltige Entwicklung" hat sich seit dem ausgehenden 20. Jahrhundert als das maßgebliche internationale Leitmotiv von Umweltpolitik und Ressourcenmanagement durchgesetzt. Die Ergebnisse des gegenständlichen Projekts sollen einen Beitrag zur Umsetzung der Ziele einer umfassenden nachhaltigen Entwicklung leisten, wie sie bei der Umwelt- und Entwicklungskonferenz der Vereinten Nationen in Rio de Janeiro 1992 (UNCED, United Nations Conference on Environment and Development) und nachfolgenden Prozessen, wie der Ministerkonferenz zum Schutz der Wälder in Europa (MCPFE, Ministerial Conference on the Protection of Forests in Europe), festgeschrieben worden sind. Die nachhaltige Nutzung der Komponenten der biologischen Vielfalt ist außerdem eines der drei erklärten Ziele des Übereinkommens über die biologische Vielfalt (CBD, Convention on Biological Diversity). Das Übereinkommen zielt im Wesentlichen auf die Erhaltung der biologischen Vielfalt der Ökosysteme, der Arten und Populationen sowie deren natürlicher genetischer Differenzierung ab, wobei ein Gleichgewicht zwischen Schutz und nachhaltiger Nutzung der biologischen Vielfalt angestrebt wird.

Übergeordnete internationale Prinzipien für nachhaltige Nutzung, die grundsätzliche politische Richtlinien auch für nachhaltige Wildtiernutzung zur Verfügung stellen, ergeben sich aus dem Übereinkommen über die biologische Vielfalt (CBD), insbesondere dem Ecosystem Approach (UNEP, 2000) und den Addis Abeba Principles and Guidelines (UNEP, 2004), sowie den Erklärungen der IUCN $(2000,2001)$ und der European Charter on Hunting and Biodiversity (Council of Europe, 2007). Für die konkrete Umsetzung der Nachhaltigkeits-Vision in die Praxis der verschiedenen Landnutzungsformen sind jedoch operationale Umsetzungsrichtlinien und entsprechende Instrumente für die Bewertung, das Monitoring und für ein adaptives Management erforderlich. Dazu werden auf internationaler Ebene in verschiedenen Fachbereichen Richtlinien, Prinzipien, Kriterien, Indikatoren, Standards und Zertifizierungssysteme entwickelt und als Steuerungsinstrumente für die Entwicklung von nachhaltiger Nutzung angewandt.

Das vorliegende Projekt baut auf den oben angeführten umweltpolitischen Übereinkommen und den nachfolgenden Entwicklungsschritten, die sich daraus ergeben haben, auf. Die Zielsetzung des Projekts entspricht insbesondere auch der Grundsatzerklärung zur nachhaltigen Nutzung wild lebender Ressourcen, die beim Weltkongress der International Union for Conservation of Nature and Natural Resources (Weltnaturschutzunion / IUCN) in Amman/Jordanien im Jahr 2000 beschlossen wurde (IUCN, 2000). In der Resolution der IUCN wird festgestellt, dass auch die Nutzung von wild lebender Fauna und Flora, sofern sie nachhaltig erfolgt, ein Instrument des Naturschutzes sein kann und zur Erhaltung der biologischen Vielfalt beitragen kann. Dies trifft auch auf die Jagd zu. Ausgenommen sind Schutz- 
gebiete, wie Wildnisgebiete, Nationalparks etc., in denen eine entnehmende Nutzung definitionsgemäß ganz oder auf Teilflächen nicht zulässig ist. Weiters sollen durch die vorliegende Arbeit auch grundlegende Beiträge zur Umsetzung von Zielen der Alpenkonvention erbracht werden, die etwa in den Protokollen der Bereiche Naturschutz und Landschaftspflege, Raumplanung und nachhaltige Entwicklung, Bergwald und Tourismus enthalten sind.

Eine Entwicklung kann als nachhaltig bezeichnet werden, wenn sie den Lebensbedürfnissen gegenwärtiger Generationen gerecht wird, ohne die Möglichkeit zukünftiger Generationen zu gefährden, ihre eigenen Bedürfnisse befriedigen zu können (Brundtland \& UNCED, 1988). In allgemeiner Form kann eine „nachhaltige Nutzung“ natürlicher Ressourcen als eine Form und Intensität der Nutzung definiert werden, welche

- einen Ausgleich zwischen Schutz und Nutzung anstrebt;

- die Grenzen der ökologischen Tragfähigkeit und der Funktionsfähigkeit der Ökosysteme beachtet;

- die Regenerationsfähigkeit erneuerbarer biologischer Ressourcen nicht überschreitet;

- sozial gerecht und ausgewogen ist;

- die qualitativ und quantitativ gleichwertige Nutzung von Ressourcen sowohl jetzt als auch für zukünftige Generationen ermöglicht.

Aus ökologischer Sicht erfordert nachhaltige Nutzung insbesondere, dass durch menschliches Handeln die globalen Stoffkreisläufe nicht irreversibel beeinträchtigt und lokale Belastbarkeitsgrenzen der Ökosysteme nicht überschritten werden dürfen. Eine nachhaltige Nutzung der natürlichen Ressourcen muss sich der Erhaltung der ökologischen Funktionsfähigkeit unterordnen, um die dauerhafte und gleichmäßige Erfüllung aller materiellen und immateriellen Leistungen und Funktionen der natürlichen Umwelt zu gewährleisten. Eine ökologisch nachhaltige Jagd darf sich nicht auf die jagdliche Abschöpfung des maximalen nachhaltig nutzbaren Wildzuwachses beschränken, sondern es ist ebenso eine Vielzahl qualitativer Aspekte zu berücksichtigen. So müssen insbesondere die Vielfalt der Arten, Populationen und der genetischen Variabilität, aber auch der Lebensräume und des Landschaftsbildes erhalten werden. Auch Österreich bekennt sich dazu, die anerkannten Grundsätze der ökologischen, sozialen und wirtschaftlichen Nachhaltigkeit in alle Bereiche der Gesellschafts- und Wirtschaftspolitik und alle Ebenen der Entscheidungsfindung zu integrieren (BMU, 1995; Österreichische Bundesregierung, 2002).

Entsprechend den „drei Säulen“ der Nachhaltigkeit soll die vorliegende Arbeit neben der ökologischen auch die ökonomische und sozio-kulturelle Komponente berücksichtigen. Grundsätzlich sollen etwa die ökonomische Ertragsfähigkeit der Jagd erhalten, aber auch etwaige Schäden, die von der Jagdbewirtschaftung herrühren, vermieden werden. Wesentlich ist beispielsweise auch, dass die Jagd den Intentionen eines zeitgemäßen Tierschutzes entgegenkommt. Beitrag und Verantwortungsbereitschaft der Jagd zur nachhaltigen gesellschaftlichen Entwicklung sollen aus dem kulturellen jagdlichen Selbstverständnis ersichtlich sein. Dies gilt ebenso für das jeweilige Selbstverständnis anderer Landnutzergruppen, die Wildtiere und Jagd beeinflussen.

Kriterien- und Indikatorensysteme stellen anerkannte Bewertungsinstrumente dar, die es ermöglichen, die Nachhaltigkeit von Nutzungen und das Erreichen von Nachhaltigkeitszielen zu überprüfen. Derartige Bewertungsansätze wurden bereits für die Anwendung in verschiedenen Nutzungssektoren entwickelt, wie zum Beispiel in der Forstwirtschaft, der Landwirtschaft oder der Fischerei. Für den Sektor der Jagd wurde die diesbezüglich bestehende Lücke durch die Vorarbeiten zum gegenständlichen Projekt geschlossen (Forstner et al., 2001, 2003, 2006). Nachhaltige Nutzung von Wildtierlebensräumen und Wildtieren kann aber letztlich nur gelingen, wenn alle im Wildlebensraum agierenden Landnutzergruppen sich der Auswirkungen ihrer Tätigkeiten auf Wildtierressourcen sowie auf die jeweils anderen Nutzergruppen bewusst sind, und wenn die Nachhaltigkeitsansprüche anderer Nutzergruppen in 
der jeweils eigenen Landnutzungspraxis bestmöglich berücksichtigt werden. Dafür bedarf es integrativer, intersektoral abgestimmter Nachhaltigkeitsansätze für eine gesamtnachhaltige Landnutzung sowie deren Operationalisierung für die Anwendung auf regionaler Ebene. Bislang existieren in der Praxis jedoch kaum sektorübergreifende Bewertungsinstrumente für nachhaltige Nutzung (Hartje et al., 2003). Auch Methoden für die Entwicklung sektorübergreifender Kriterien und Indikatoren sind bisher nicht etabliert (Linser, 2001).

Deshalb musste zuerst ein geeignetes Konzept für die methodische Vorgehensweise bei der Ausarbeitung des Bewertungsinstrumentariums erstellt werden. Aufbauend auf den erwähnten Vorarbeiten und in enger Abstimmung mit Vertretern der betroffenen Interessengruppen wurde dann ein transparentes und weitest möglich objektiviertes Bewertungssystem partizipativ mit den potenziellen Anwendern entwickelt, das es nun unterschiedlichen Landnutzern ermöglicht, den Nachhaltigkeitsgrad ihrer eigenen Tätigkeit im Hinblick auf Wildtiere, Wildtierlebensräume und Jagd selbst zu überprüfen. Die vorliegenden Prinzipien, Kriterien und Indikatoren für integratives nachhaltiges Wildtiermanagement wurden als freiwillig anzuwendende Hilfe zur Selbsteinschätzung konzipiert. Sie sollen die eigene Standortbestimmung im Hinblick auf den Nachhaltigkeitsbeitrag zum gegenständlichen Thema unterstützen und zum Hinterfragen der eigenen Handlungspraxis anregen.

\subsection{Projektstruktur}

Zur Erreichung der Projektziele war das Projekt ISWI-MAB in den nachstehend aufgeführten sechs Arbeitspaketen organisiert.

Tabelle 1: Die Arbeitspakete von ISWI-MAB.

\begin{tabular}{|l|l|}
\hline AP Nr. & Bezeichnung Arbeitspaket \\
\hline AP1 & Projektmanagement \\
\hline AP2 & Stakeholder-Partizipation \\
\hline AP3 & Nachhaltiges jagdliches Wildtiermanagement \\
\hline AP4 & Bewertungsmethodik \\
\hline AP5 & $\begin{array}{l}\text { Sektorübergreifende Bewertung von Schnittstellen } \\
\text { (Wildtiere / Habitate - Jagd - Landnutzungen) }\end{array}$ \\
\hline AP6 & Konsequenzen für Management und Monitoring \\
\hline
\end{tabular}

Die Projektstruktur (Arbeitspakete, Ablauf und Zusammenspiel, Input-Output-Flüsse, iterative Arbeitsschleifen, etc.) ist in der nachstehenden Abbildung (Abbildung 1) visualisiert. 

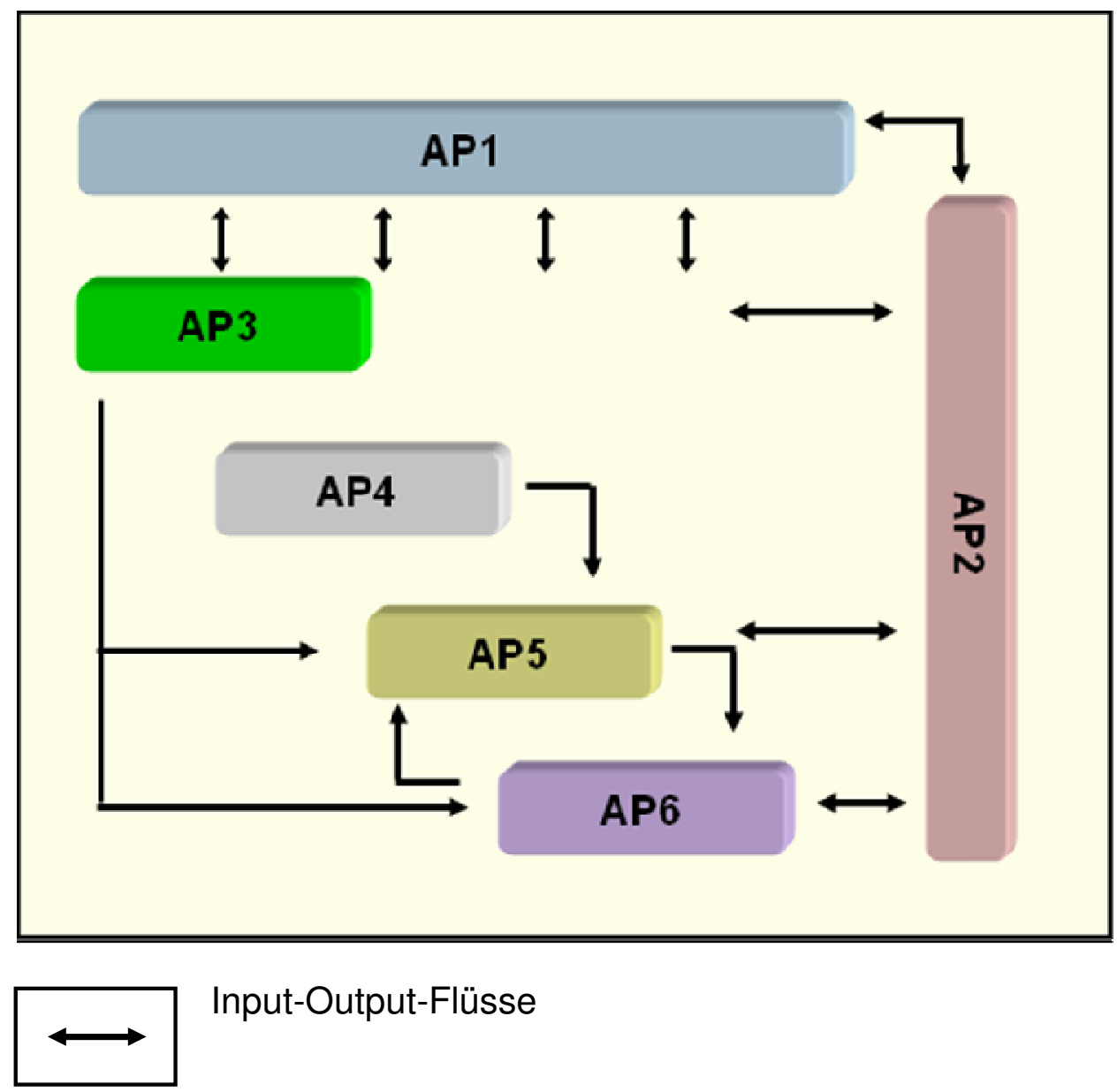

\section{Arbeitspakete:}

$\square$ AP1: Projektmanagement

$\square$ AP2: Stakeholder-Partizipation

$\square$ AP3: Nachhaltiges jagdliches Wildtiermanagement

$\square$ AP4: Bewertungsmethodik

AP5: Sektorübergreifende Bewertung von Schnittstellen (Wildtiere / Habitate - Jagd Landnutzungen)

AP6: Konsequenzen für Management und Monitoring

Abbildung 1: Projektarchitektur und wesentliche Informationsflüsse zwischen Arbeitspaketen. 


\subsection{Aufbau des Berichts}

Die Projektergebnisse werden im Hauptteil des Endberichts (Kapitel 4, Kapitel 5) sowie im Anhang (Kapitel 8) präsentiert. Folgende Hauptergebnisse werden in den angegebenen Kapiteln vorgelegt:

- Intersektorale Bewertungssets für integratives nachhaltiges Wildtiermanagement im Biosphärenpark Wienerwald (mit Prinzipien, Kriterien, Indikatoren, umfangreichen Erläuterungen und Anwendungshinweisen) für die Nutzergruppen Jagd, Forstwirtschaft, Landwirtschaft und Freizeit- und Erholungsmanagement

- Voll- und Kurzversionen (vollständige Fassungen) (Kapitel 8.1: Anhang - Ergebnisse)

- Überblickstabellen (Vollversionen) (Kapitel 4.3.2)

- Überblickstabellen (Kurzversionen) (Kapitel 5.1)

- Ergebnisse der Experteninterviews (Kapitel 4.1.1)

- Ergebnisse der Nutzerbefragungen (Kapitel 4.1.2)

- Sammlungen der intersektoralen Schnittstellen-Themen (Kapitel 4.2)

- Empfehlungen für integratives Habitat- und Wildtiermanagement (Kapitel 5)

- Verknüpfung von Maßnahmenbereichen mit den Bewertungssets (Kapitel 5.2)

- Regelwerke, Konzepte und Instrumente im Biosphärenpark und Hinweise zur Umsetzung von Projektinhalten (Kapitel 5.3)

- Empfehlungen für das Management in Kernzonen (für Jagd und das Freizeit- und Erholungsmanagement) (Kapitel 5.4) 